\title{
Impaired Long-Term Memory and NR2A-Type NMDA Receptor-Dependent Synaptic Plasticity in Mice Lacking c-Fos in the CNS
}

\author{
Alexander Fleischmann, ${ }^{1}$ Oivind Hvalby, ${ }^{2}$ Vidar Jensen, ${ }^{2}$ Tatyana Strekalova, ${ }^{3}$ Christiane Zacher, ${ }^{3}$ Liliana E. Layer, ${ }^{4}$ \\ Ane Kvello, ${ }^{2}$ Markus Reschke, ${ }^{1}$ Rainer Spanagel, ${ }^{3}$ Rolf Sprengel, ${ }^{4}$ Erwin F. Wagner, ${ }^{1}$ and Peter Gass ${ }^{3}$ \\ ${ }^{1}$ Research Institute of Molecular Pathology, 1030 Vienna, Austria, ${ }^{2}$ Institute of Basic Medical Sciences, University of Oslo, N-0317 Oslo, Norway, ${ }^{3}$ Central \\ Institute of Mental Health, D-68159 Mannheim, Germany, and ${ }^{4}$ Max Planck Institute for Medical Research, D-69120 Heidelberg, Germany
}

The immediate early gene c-fos is part of the activator protein-1 transcription factor and has been postulated to participate in the molecular mechanisms of learning and memory. To test this hypothesis in vivo, we generated mice with a nervous system-specific c-fos knock-out using the Cre-loxP system. Adult mice lacking c-Fos in the CNS (c-fos ${ }^{\Delta \mathrm{CNS}}$ ) showed normal general and emotional behavior but were specifically impaired in hippocampus-dependent spatial and associative learning tasks. These learning deficits correlated with a reduction of long-term potentiation (LTP) in hippocampal CA3-CA1 synapses. The magnitude of LTP was restored by a repeated tetanization procedure, suggesting impaired LTP induction in c-fos ${ }^{\Delta \mathrm{CNS}}$ mice. This rescue was blocked by a selective inhibitor of NR2Btype NMDA receptors. This blockade was compensated in wild-type mice by NR2A-type NMDA receptor-activated signaling pathways, thus indicating that these pathways are compromised in c-fos ${ }^{\Delta \mathrm{CNS}}$ mice. In summary, our data suggest a role for c-Fos in hippocampusdependent learning and memory as well as in NMDA receptor-dependent LTP formation.

Key words: c-fos; NMDA receptor; mice; conditional; behavior; long-term potentiation; hippocampus

\section{Introduction}

Long-term learning and memory require long-lasting changes in the strength of specific synaptic connections between neurons (Martin et al., 2000; Kandel, 2001). These adaptive changes in synaptic efficacy are initiated by activity-dependent release of neurotransmitters from presynaptic nerve terminals and binding to their cognate postsynaptic receptors. In glutamatergic synapses of the hippocampus, many of the activity-dependent synaptic modifications require the participation of NMDA receptors, which are heteromultimeric ion channels composed of the NR1 subunit and one or more of the NR2A or NR2B subunits (Hollmann and Heinemann, 1994). NMDA receptor-dependent $\mathrm{Ca}^{2+}$ influx activates a cascade of biochemical events that lead to the activation of so-called immediate early genes (IEGs). IEGs regulate delayed onset of effector genes, coding for structural, growth-related, and synaptic proteins (Herdegen and Leah, 1998). A candidate molecule suggested to participate in learning and memory is the IEG c-fos (Tischmeyer and Grimm, 1999; Guzowski, 2002). c-Fos is part of a family of transcription factors

Received April 7, 2003; revised July 18, 2003; accepted July 24, 2003.

This work was supported by Grant GA 427/4-2 from the Deutsche Forschungsgemeinschaft to P.G. The Institute of Molecular Pathology is funded by Boehringer Ingelheim. We thank M. Reschke and Dr. L. Kenner for help with histology, C. Theussel and Dr. A. Bichl for maintaining our mouse colony, Drs. L. Bakiri and M. Yaniv for anti-c-Fos antibody, and Drs. P. Andersen, D. Bartsch, D. Reisel, N. Shah, and R. Wu for critically reading this manuscript.

Correspondence should be addressed to Dr. Peter Gass, Central Institute of Mental Health Mannheim, J 5 , D-68159 Mannheim, Germany. E-mail: gass@as200.zi-mannheim.de.

A. Fleischmann's present address: Columbia University, Center for Neurobiology and Behavior, 701 West 168th Street, New York, NY 10032.

Copyright $\odot 2003$ Society for Neuroscience $\quad$ 0270-6474/03/239116-07\$15.00/0 consisting of c-Fos, FosB, and the Fos-related antigens 1 and 2 (Fra-1 and Fra-2) (Herdegen and Leah, 1998). Fos proteins form heterodimeric complexes with Jun proteins to constitute the transcription factor activator protein-1 (AP-1), which regulates gene expression by binding to the $\mathrm{AP}-1$ recognition sequence found in a variety of target genes (Muller et al., 1983; Greenberg and Ziff, 1984; Chiu et al., 1988; Halazonetis et al., 1988; Kouzarides and Ziff, 1988).

First indications for a role of c-Fos during memory formation came from expression studies in which c-fos induction was observed during behavioral training and learning tasks (Tischmeyer and Grimm, 1999; Guzowski, 2002). However, because c-Fos is induced by a plethora of physiological and pathological stimuli, the functional relevance of these results was equivocal (Herdegen and Leah, 1998). The role of c-Fos for learning and memory was also studied in conventional c-fos knock-out mice (Johnson et al., 1992; Paylor et al., 1994). Because these animals suffer from developmental malformations, it was not possible to discriminate putative mnemonic impairments from confounding complex motor and other dysfunctions (Paylor et al., 1994).

To overcome these problems, we generated mice with a CNSspecific knock-out of the $\mathrm{c}$-fos gene (c-fos $\left.{ }^{\Delta \mathrm{CNS}}\right)$ using the CreloxP recombination system. We show that $c-f o s^{\Delta \mathrm{CNS}}$ mice have selective deficits in hippocampus-dependent learning and a reduced magnitude of long-term potentiation (LTP) at hippocampal CA3-to-CA1 synapses. Because NMDA receptors are necessary for LTP induction at CA1 synapses (Nicoll et al., 1988; Tsien et al., 1996), we examined the expression of NMDA receptor 
subunits and specifically the involvement of NR2A and NR2B subunits during LTP development. Pharmacological experiments with the subtype-specific NMDA receptor antagonist (1S,2S)-1-(4hydroxyphenyl)-2-(4-hydroxy-4-phenylpiperidino)-1-propanol (CP-101,606) suggest an involvement of c-Fos in NR2A-type receptor signaling pathways.

\section{Materials and Methods}

Generation of mice. The targeting construct was generated by insertion of the 5' loxP site into the EcoRI site in the first intron and a PGKneo cassette flanked by flip recombination target sites and the $3^{\prime}$ loxP site into the BamHI site downstream of the polyadenylation signal. A splice acceptor was cloned $5^{\prime}$ of the gene encoding the enhanced green fluorescent protein (EGFP) and inserted downstream of the PGKneo cassette followed by the diphtheria toxin gene to select against nonhomologous integration. The linearized construct was electroporated into E14.1 stem cells and after G415 selection, homologous recombinants were identified by PCR and Southern blot analysis. Chimeric mice were generated by blastocyst injection and transmitted the targeted allele to their offspring. The flirted PGKneo cassette was deleted in vivo using flip recombinase transgenic mice (Dymecki, 1996). Expression of the Cre recombinase results in the deletion of exons $2-4$ of $\mathrm{c}$-fos, including the dimerization and DNA binding and the transactivation domains. The deleted locus expresses the EGFP protein fused to the first exon of c-fos under the control of the c-fos promoter.

Histological analysis. EGFP expression in mouse embryos was analyzed directly under UV light. Expression of c-Fos and EGFP in the brain was induced by an injection of kainic acid $(20 \mathrm{mg} / \mathrm{kg}$, i.p.) in 3- to 6-monthold mice. Brains were dissected $3 \mathrm{hr}$ after injection, fixed in $4 \%$ paraformaldehyde, and embedded in optimal cutting temperature compound. Frozen sections were stained using anti-EGFP (1:1000; Clontech, Franklin Lakes, NJ) and anti-c-Fos (a gift from Dr. M. Yaniv, Institute Pasteur, Paris, France) antibodies according to standard procedures.

Behavioral studies. All experiments were performed with 3- to 6-month-old male mice in a mixed 129/Ola-C57BL/6 genetic background. Eighteen c-fos ${ }^{\Delta \mathrm{CNS}}$ mice and 19 control littermates (c-fos ${ }^{\mathrm{f} / \mathrm{f}}$; i.e., without a nestin-Cre transgene) were investigated. The presence of the nestin-Cre transgene in the absence of floxed c-fos alleles did not have any influence on the behavioral parameters tested (our pilot experiments) (Tronche et al., 1999). Mice were kept on a $12 \mathrm{hr}$ reversed light/dark cycle with light shifts at 6:00 A.M. and 6:00 P.M. Experiments were done during the animals' active phase. Locomotor studies were performed in activity boxes $(26 \times 26 \times 39 \mathrm{~cm}$; TrueScan System; Coulbourn, Allentown, PA). Locomotion was measured by photocells for periods of 10 min at two phases of circadian activity, respectively (i.e., $2 \mathrm{hr}$ after onset of light and $2 \mathrm{hr}$ after onset of darkness). The rotarod task consisted of one trial during which the animals were placed on a rod with increasing rotation speed (diameter, $3 \mathrm{~cm}$; starting speed, $2.5 \mathrm{rpm}$ ). Time was measured until the animals fell off the rod. Elevated zero-maze and darklight box tests were performed as described previously (Timpl et al., 1998; Tronche et al., 1999). In both tests, the animals' behavior was recorded for 5 min using a video camera system. The latencies to visit the aversive maze compartments were analyzed, as well as the number of visits and the time spent in these compartments. The water maze consists of a white circular pool of $150 \mathrm{~cm}$ diameter filled with water $\left(24^{\circ} \mathrm{C}\right)$ as described previously (Gass et al., 1998). A $14 \times 14 \mathrm{~cm}^{2}$ platform was placed invisibly $1 \mathrm{~cm}$ below the water surface at $35 \mathrm{~cm}$ from the wall of the pool. Mice were trained by one swimming trial per day on 11 consecutive days, and the maximal trial duration was $120 \mathrm{sec}$. The probe trial (free swimming without platform in the pool) was done on day 12 . The swim paths of the mice were recorded by a video camera suspended above the center of the pool and were fed to an electronic imaging system (EthoVision 1.90; Noldus, Wageningen, the Netherlands). Analysis of acquisition was done offline by extracting from digitally stored swim paths a number of parameters characterizing water maze learning in mice. Presented here are the length of swim paths, the percentage of time spent either in the target quadrant or in three control quadrants, and the swimming speed. Fear conditioning was done as described previously (Gass et al., 1998). For both contextual and cued conditioning, mice were individually placed into the conditioning chamber $\left(58 \times 30 \times 27 \mathrm{~cm}^{3}\right.$; TSE, Bad Homburg, Germany) and allowed to habituate for $2 \mathrm{~min}$ before the onset of a discrete conditioned stimulus $(2800 \mathrm{~Hz}$ tone; $85 \mathrm{~dB}$ ) that lasted $30 \mathrm{sec}$. At the end of the tone, animals were subjected to the unconditioned stimulus (continuous footshock of $0.7 \mathrm{~mA}$ for $3 \mathrm{sec}$ ). Twenty-four hours after training, context conditioning was assessed by measuring freezing, defined as a complete lack of movement other than respiration. Context learning was tested in the same Plexiglas chamber that was used for training by measuring freezing for $5 \mathrm{~min}$. Cued conditioning was analyzed in a novel context at $48 \mathrm{hr}$ after training by exposing the animals to the tone for $3 \mathrm{~min}$, during which freezing was scored. Statistical analysis was performed by repeated two-way ANOVA and planned post hoc Scheffe comparisons, or by Student's $t$ test in combination with the Bonferroni-Dunn correction, respectively. Errors indicated are SEM.

Electrophysiological studies. Four- to 6-month-old mutant and control mice were killed with halothane. Brains were removed and cooled to $0-4^{\circ} \mathrm{C}$ in artificial CSF (ACSF) of the following composition (in $\mathrm{mm}$ ): $124 \mathrm{NaCl}, 2 \mathrm{KCl}, 1.25 \mathrm{KH}_{2} \mathrm{PO}_{4}, 2 \mathrm{MgSO}_{4}, 1 \mathrm{CaCl}_{2}, 26 \mathrm{NaHCO}_{3}$, and 12 glucose, bubbled with $95 \% \mathrm{O}_{2}-5 \% \mathrm{CO}_{2}, \mathrm{pH}$ 7.4. Transverse slices (400 $\mu \mathrm{m})$ from the middle portion of each hippocampus were cut with a vibroslicer in $4^{\circ} \mathrm{C} \mathrm{O}_{2}-\mathrm{CO}_{2}$-bubbled ACSF. Slices were placed in an interface chamber exposed to humidified gas at $28-32^{\circ} \mathrm{C}$ and perfused with ACSF containing $2 \mathrm{mM} \mathrm{CaCl}_{2}$. Orthodromic synaptic stimuli $(50 \mu \mathrm{sec}$, $<150 \mu \mathrm{A}, 0.1 \mathrm{~Hz}$ ) were delivered alternately through two tungsten electrodes: one in the stratum radiatum and the other in the stratum oriens of the CA1 region. Extracellular synaptic responses were monitored by two glass electrodes (filled with ACSF) placed in the corresponding synaptic layers. After stable synaptic responses had been obtained in both pathways for at least $15 \mathrm{~min}$, one pathway was tetanized (either with a single $100 \mathrm{~Hz}$ tetanization for $1 \mathrm{sec}$ or with four such tetanizations given at 5 min intervals). To standardize, the stimulation strength used for tetanization was just above threshold for generation of a population spike in response to a single test shock. The synaptic efficacy was assessed by measuring the slope of the field EPSP in the middle third of its rising phase. Six consecutive responses (1 min each) were averaged and normalized to the mean value recorded 1-4 min before tetanization. The untetanized pathway served as the control pathway and was recorded simultaneously. The oriens and radiatum pathways were tetanized equally as often. The magnitude of LTP was not different in the oriens and the radiatum pathways and was always specific for the tetanized pathway. Data were pooled across animals of the same age and genotype and are presented as mean \pm SEM. Statistical significance was evaluated using a two-tailed $t$ test. In some experiments, $50 \mu \mathrm{M}$ DL-2-amino-5phosphonopentanoic acid (DL-AP-5) was present during the recordings. The concentration of CP-101,606 (10 $\mu \mathrm{M})$ used in a subset of experiments has been reported to selectively reduce NR2B-mediated currents in slices (Stocca and Vicini, 1998). Furthermore, paired-pulse responses (mean of five trials at nine different stimulation strengths at 20,50, 100, and $200 \mathrm{msec}$ interstimulus interval) were monitored. The maximal slope of the field EPSP was measured and the facilitation ratio was calculated as EPSP2 slope/EPSP1 slope. To pool data from different experiments, we selected responses to a stimulation strength just below threshold for eliciting a population spike on the second EPSP.

NMDA receptor subunit expression studies. Whole hippocampi were homogenized in $25 \mathrm{~mm}$ HEPES, $\mathrm{pH}$ 7.4, including protease inhibitor mixture (Complete; Roche, Mannheim, Germany) and centrifuged for 5 min at $2000 \mathrm{rpm}$ and $4^{\circ} \mathrm{C}$ to remove debris and nuclei. For each sample, $12 \mu \mathrm{g}$ of total protein determined by BCA (protein assay kit; Pierce, Rockford, IL) were separated on 8\% SDS-polyacrylamide gels and transferred to nitrocellulose membranes. The blotted proteins were probed with the following antibodies: polyclonal anti-glutamate receptor A (GluRA) $(0.05 \mu \mathrm{g} / \mathrm{ml}$; Chemicon, Temecula, CA), polyclonal anti-NR1 $(0.17 \mu \mathrm{g} / \mathrm{ml}$; Chemicon), polyclonal anti-NR2A $(0.33 \mu \mathrm{g} / \mathrm{ml}$; Novus, Littleton, CO), polyclonal anti-NR2B $(0.2 \mu \mathrm{g} / \mathrm{ml}$; Novus $)$, monoclonal anti- $\beta$-actin (clone AC-15, 1:40,000; Sigma, St. Louis, MO) and horseradish peroxidase-linked goat anti-rabbit or anti-mouse secondary antibodies (1:15,000; Jackson ImmunoResearch, West Grove, PA). For visu- 
alization, the enhanced chemiluminescence method (ECL-Plus; Amersham Biosciences, Freiburg, Germany) was used.

\section{Results}

Generation of mice with a CNS-specific c-fos deletion

Mice carrying a floxed c-fos allele were generated by inserting loxP sites into the first intron and downstream of the polyadenylation signal of the c-fos gene (Fig. $1 \mathrm{~A}$ ). In addition, EGFP was inserted downstream of the $3^{\prime}$ loxP site so that when $\mathrm{c}$-fos is deleted, EGFP is expressed by the c-fos promoter. Homozygous mice carrying the floxed allele $\left(\mathrm{c}-f_{0 s}{ }^{\mathrm{f} / \mathrm{f}}\right.$ ) were phenotypically normal. In contrast, ubiquitous deletion of the floxed allele $\left(c-f o s^{\Delta / \Delta}\right)$ resulted in a phenotype indistinguishable from conventional c-fos knock-out mice, exhibiting growth retardation and osteopetrosis (data not shown). In heterozygous c- $f o s^{\Delta /+}$ mice, EGFP expression correlated with sites of c-fos expression during development (e.g., in developing bones, in hair follicles, and interdigitally) (Fig. $1 B$ ).

Mice carrying the floxed c-fos allele were then crossed with nestin-Cre transgenic mice, which express the Cre recombinase in all neural cells of the CNS, including neurons and glia (Tronche et al., 1999). Efficient and CNS-specific deletion of the c-fos gene was detected by Southern blot analysis of genomic DNA prepared from various brain regions and nonneuronal tissues of 3- to 6-month-old mice (data not shown). When c-fos ${ }^{\mathrm{f} / \mathrm{f}}$ mice were injected with kainic acid, a strong inducer of c-Fos activity, c-Fos immunoreactivity was observed in several regions of the brain, including the hippocampus, the cortex, and the amygdala. In contrast, no c-Fos-positive cells were seen in $c-f o s ~ f / f$ mice carrying the nestin-Cre transgene (cfos ${ }^{\Delta \mathrm{CNS}}$ mice) (Fig. 1C). In c-fos ${ }^{\Delta \mathrm{CNS}}$ mice, injection of kainic acid induced EGFP expression in neurons (Fig. 1C). Furthermore, the spatial and temporal pattern of EGFP induction closely correlated with the pattern of c-Fos induction (data not shown). Thus, we conclude that the floxed c-fos allele is functional, that c-fos ${ }^{\Delta \mathrm{CNS}}$ mice lack c-Fos expression in the CNS, and finally, that the EGFP reporter gene recapitulates the expression pattern of endogenous c-Fos.

General, locomotor-related, and anxiety-related behaviors are normal in c-fos ${ }^{\Delta \mathrm{CNS}}$ mice

Consistent with previous reports using conventional c-fos knockout mice (Johnson et al., 1992; Wang et al., 1992; Watanabe et al., 1996), histological analyses revealed no gross developmental abnormalities in the CNS structure in general and in the hippocampus of $\mathrm{c}-\mathrm{fos}^{\Delta \mathrm{CNS}}$ mice compared with $\mathrm{c}$-fos ${ }^{\mathrm{f} / \mathrm{f}}$ and wild-type controls (data not shown). c-fos ${ }^{\Delta \mathrm{CNS}}$ mice grew and mated normally, and their overall behavior was indistinguishable from that of

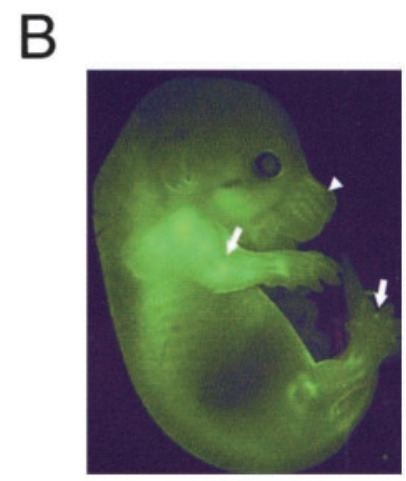

4... loxp site I... frt site
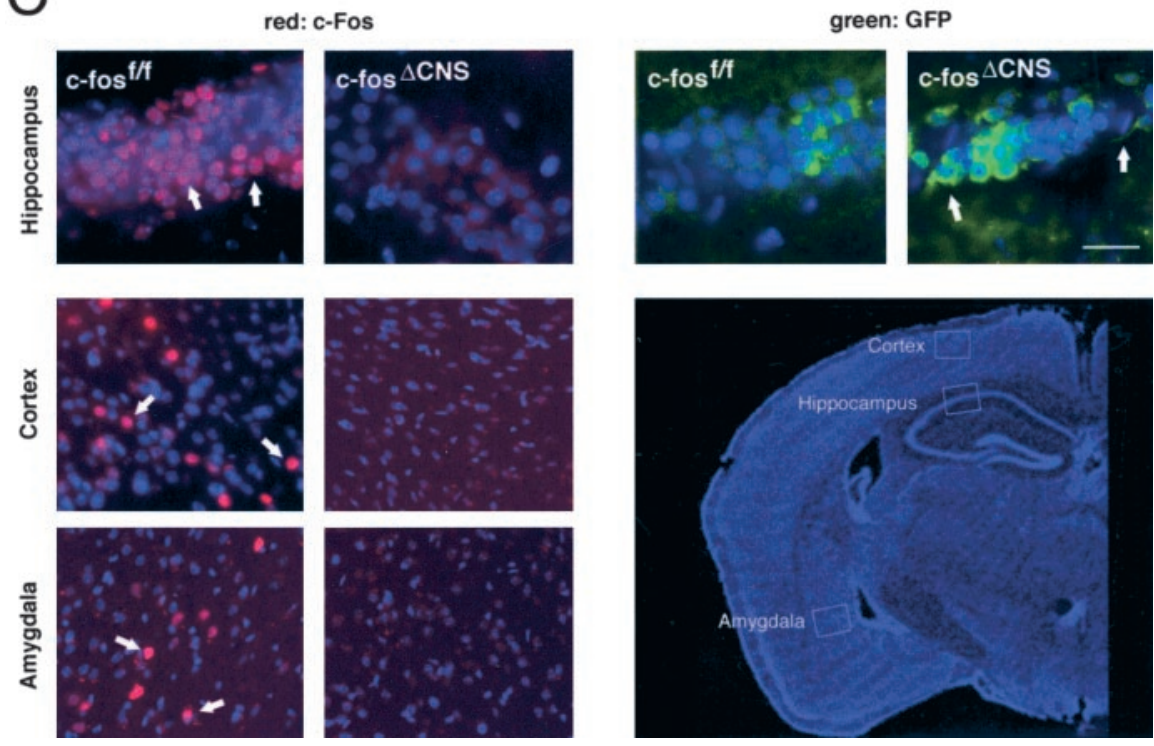

Figure 1. Generation of mice deficient for c-fos in the nervous system. A, Targeting strategy: structure of the wild-type c-fos locus, the targeted locus after recombination in embryonic stem cells, and the floxed allele after Flp-mediated deletion of the promoter. Exons (E1-E4) are in boxes, coding areas are in black, and pA indicates the transcriptional stop of the c-fos gene. $B$ Developmental expression (E14.5) of the EGFP reporter in heterozygous c-fos ${ }^{\Delta /+}$ mice. Arrows indicate expression in bones and

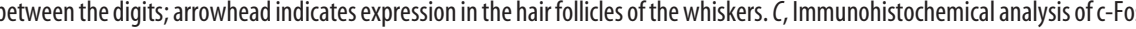
expression in the hippocampus, cortex, and amygdala of $c-f o s^{f / f}$ and $c-f o s{ }^{\Delta \mathrm{CNS}}$ mice (left). After intraperitoneal kainic acid labeling revealed cytoplasmic EGFP expression in $c-f o s{ }^{\Delta \mathrm{CNS}}$ but not in $\mathrm{c-fos}{ }^{\mathrm{f} / \mathrm{f}}$ mice (right). The white rectangles in the lowmagnification image (bottom right) indicate the areas selected for immunohistochemistry. Scale bar, $50 \mu \mathrm{m}$.

their littermates. More detailed behavioral tests on 3- to 6-month-old c-fos ${ }^{\Delta \mathrm{CNS}}$ mice revealed regular horizontal and vertical locomotion in activity boxes (Fig. $2 A$ ) and normal muscular strength and motor skills when tested on a rotarod task (Fig. $2 B$ ) and during swimming in the water maze (Fig. 2C). c-fos ${ }^{\Delta \mathrm{CNS}}$ mice exhibited normal emotional behavior: in the dark-light box, they had similar latencies to visit the anxiety-related light compartment, as well as similar numbers of visits and time spent in this compartment as control littermates (Fig. 2D). Furthermore, the anxiety-related scores of $c-f o s{ }^{\Delta \mathrm{CNS}}$ mice were not different from controls in the elevated zero-maze (data not shown).

\section{c-fos ${ }^{\Delta \mathrm{CNS}}$ mice show deficits in spatial learning}

We then examined the mice in the Morris water maze task, a test for spatial learning that is regarded as the model for declarative memory in rodents (Morris et al., 1982). During the acquisition phase of the test (i.e., the first $11 \mathrm{~d}$ of daily training), both mutant 


\section{locomotion:}
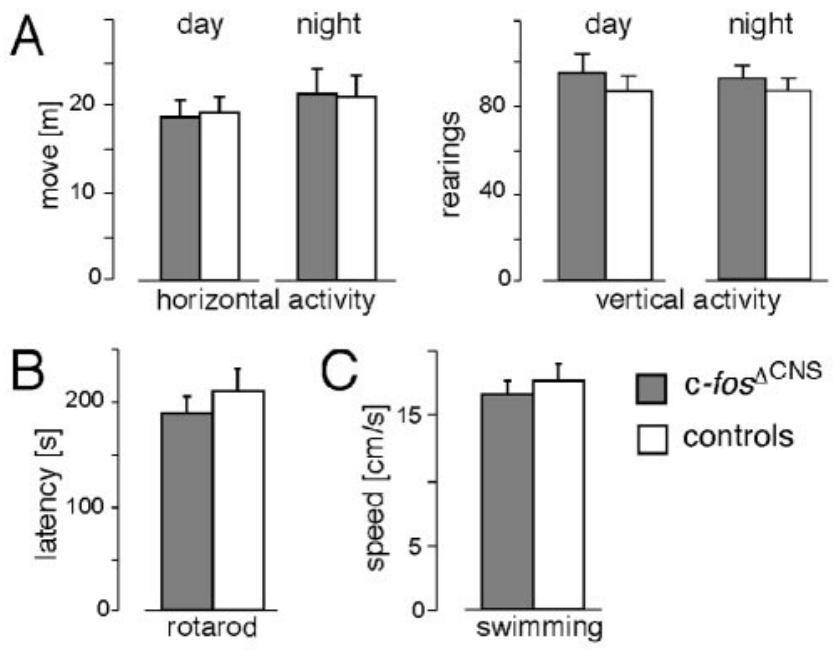

dark-light box:
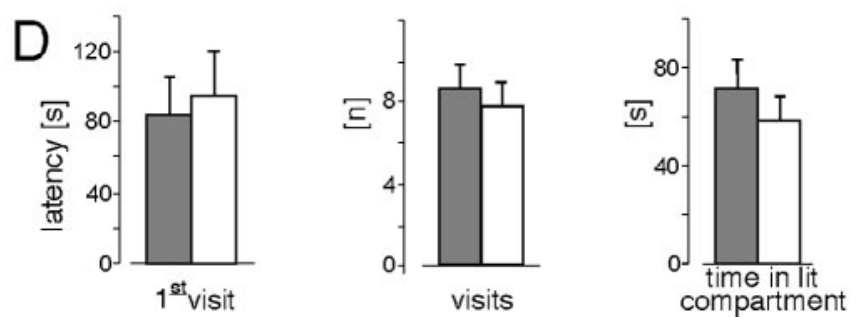

Figure 2. Normal locomotion, motor skills, and anxiety-related behaviors in c-fos ${ }^{\Delta \mathrm{CNS}}$ mice. $A$, In locomotor activity boxes, $c-$ fos ${ }^{\Delta C N S}$ mice demonstrate horizontal and vertical locomotor activity similar to that seen for control littermates during the day and night. $B, 0 \mathrm{n}$ the rotarod test, $c$-fos ${ }^{\Delta \text { CNS }}$ mice and controls show the same retention time. $C$, The Morris water maze reveals no difference between $c-$ fos ${ }^{\Delta \mathrm{CNS}}$ and control mice in swimming speed. $D$, In the darklight box test, c-fos ${ }^{\Delta \mathrm{CNS}}$ mice and control littermates have similar latencies to visit the anxietyrelated light compartment, similar numbers of visits to this compartment, and similar time spent therein.

and control mice learned to localize the hidden platform in the swimming pool, as evidenced by daily decreasing swim path length (Fig. 3A) and lower latencies to find the platform (data not shown). Thus, mutant and control mice clearly learned a strategy to escape from the water. When we removed the platform on day 12 (probe trial, a hippocampus-dependent test) (Bannerman et al., 1995), control mice spent significantly more time in the former platform quadrant $(36 \% ; p<0.01)$ than the average time in the other three quadrants (Fig. $3 B$ ). In contrast, c-fos ${ }^{\Delta C N S}$ mice spent a similar amount of time in all four quadrants (Fig. $3 B$ ). Thus, hippocampus-dependent spatial learning was disturbed in $c$ - $f \circ s^{\Delta \mathrm{CNS}}$ mice in the water maze, but the learning of the task in general was not affected.

\section{c-fos ${ }^{\Delta \mathrm{CNS}}$ mice are impaired in contextual but not in cued fear conditioning}

For additional evaluation of hippocampus-dependent learning, we subjected c-fos ${ }^{\Delta \mathrm{CNS}}$ mice to fear conditioning. Fear conditioning is an associative (Pavlovian) learning paradigm that can be tested in two different forms. The context-dependent version is dependent on both hippocampus and amygdala, whereas the cue-dependent version is reported to be exclusively amygdala dependent (Rogan and LeDoux, 1996). In both versions, the amount of freezing (immobility) represents a correlate of asso- ciative memory. Compared with control littermates, c-fos ${ }^{\Delta \mathrm{CNS}}$ mice failed to show a strong freezing response when re-exposed to the conditioning chamber $24 \mathrm{hr}$ after the training session, thus showing a significant deficit in context-dependent fear conditioning ( $18 \%$ in $c-f o s^{\Delta \mathrm{CNS}}$ mice vs $47 \%$ in controls; $p<0.01$ ) (Fig. 3C). The capability to show a freezing response was not altered, because both $c$-fos ${ }^{\Delta \mathrm{CNS}}$ and control animals demonstrated similar freezing scores in the cued version of the test (Fig. $3 C$ ). Thus, associative learning involving the hippocampus was impaired by the disruption of c-fos, although associative learning per se remained intact.

Long-term potentiation is impaired in c-fos ${ }^{\Delta \mathrm{CNS}}$ mice

To study NMDA receptor-dependent LTP of synaptic transmission, we examined LTP at hippocampal CA3-CA1 synapses. In hippocampal slices from adult control littermates, we observed robust LTP 40-45 min after a single tetanization of the afferent fibers in either the stratum radiatum or the stratum oriens (100 $\mathrm{Hz}, 1 \mathrm{sec}$ ). The average field EPSP slope was $1.46 \pm 0.05$ (mean \pm SEM) of the pretetanic control value in the tetanized pathway, whereas the synaptic transmission in the control pathway was unchanged (Fig. 4A). In contrast, we found a significant reduction of LTP in slices from $c$ - $f \circ s^{\Delta \mathrm{CNS}}$ mice $(1.27 \pm 0.05 ; p=0.01)$ (Fig. 4A). This difference was most likely caused by insufficient LTP induction in c-fos ${ }^{\Delta \mathrm{CNS}}$ mice, because LTP could be restored by four tetanic stimulations given with $5 \mathrm{~min}$ intervals (Fig. $4 B$ ). Using this stimulation protocol, the magnitude of LTP in c-fos ${ }^{\Delta \mathrm{CNS}}$ mice was comparable with LTP levels obtained in slices from control mice using the four tetanization procedure (1.67 \pm 0.09 in c-fos ${ }^{\Delta \mathrm{CNS}}$ mice vs $1.54 \pm 0.08$ in control mice) (Fig. $4 B$ ). LTP elicited by a four tetanic stimulations protocol was NMDA receptor dependent, because it was fully blocked by $50 \mu \mathrm{M}$ DLAP-5. The tetanized pathway was not significantly different from the untetanized control pathway at $40-45 \mathrm{~min}$ in slices from control mice $(n=7 ; p=0.25)$ or in slices from $c$-fos ${ }^{\Delta \mathrm{CNS}}$ mice $(n=8 ; p=0.43)$. To characterize excitatory synaptic transmission in the hippocampal CA1 region, we also measured pairedpulse facilitation (PPF) (Andersen, 1960), a short-lasting form of synaptic potentiation that is primarily attributed to changes in the presynaptic $\mathrm{Ca}^{2+}$ homeostasis (Katz and Miledi, 1968; Zucker, 1989). At four interstimulus intervals $(20,50,100$, and $200 \mathrm{msec})$, we found a slight reduction in PPF $(p<0.05)$ in the c-fos ${ }^{\Delta \mathrm{CNS}}$ mice compared with wild-type animals $(1.47 \pm 0.04 \mathrm{vs}$ $1.67 \pm 0.07,1.45 \pm 0.04$ vs $1.66 \pm 0.06,1.29 \pm 0.05$ vs $1.48 \pm$ 0.04 , and $1.14 \pm 0.02$ vs $1.21 \pm 0.03$, respectively; $n=14$ at all intervals and in both genotypes). This observation might indicate a slightly increased release probability of presynaptic glutamate in the absence of c-Fos (Murthy et al., 1997).

\section{Restoration of LTP induction in c-fos ${ }^{\Delta \mathrm{CNS}}$ mice depends on NR2B subunit-containing receptors}

The impairments of c-fos ${ }^{\Delta \mathrm{CNS}}$ mice in learning and LTP induction were similar to those described in mice with a disruption of the NMDA receptor subunit NR2A (Sakimura et al., 1995; Kiyama et al., 1998). Therefore, we investigated whether alterations in NMDA receptor expression or function could account for the deficits. In Western blot studies of hippocampal protein extracts, c-fos ${ }^{\Delta \mathrm{CNS}}$ mice revealed the same expression levels of the receptor subunits NR1, NR2A, NR2B, and GluRA as their littermate controls (Fig. 5A). However, the restoration of LTP magnitude by four repetitive tetanic stimulations in c-fos ${ }^{\Delta \mathrm{CNS}}$ mice was dependent on NR2B-type NMDA receptors. The phenylethanolamine derivative CP-101,606, a noncompetitive and selective 

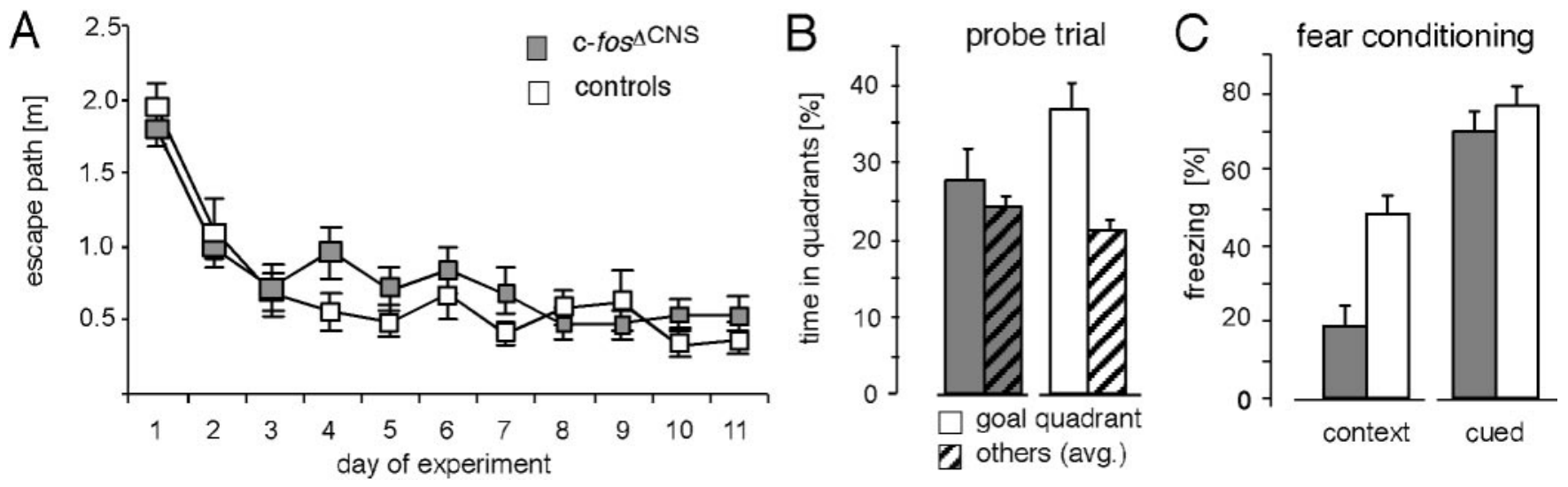

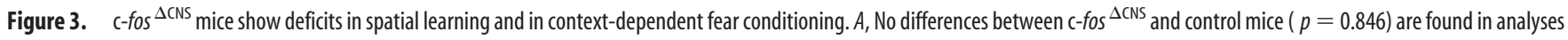
of swim path lengths needed to localize the platform during daily training. $B$, In contrast, a group comparison of probe trials by repeated two-way ANOVA exhibits a difference between $c$-fos mutants and controls $(p<0.05)$. The probe trial on day 12 demonstrates that control mice search specifically in the former goal quadrant ( $36 \%$ of time; $c$ chance level, $25 \% ; p<0.01$ ), whereas $c$-fos $\Delta$ CNS mice search randomly for the platform (avg., average). C, The freezing response on a contextual fear conditioning test $24 \mathrm{hr}$ after training is significantly reduced in c-fos $\Delta$ cNs mice ( $18 \mathrm{vs} 47 \%$ in controls; $p<0.01$ ). In cued (tone) conditioning, however, c-fos ${ }^{\Delta \mathrm{CNS}}$ mice and control littermates have similar scores (70 vs $77 \%$; not significant).

NR2B blocker (Williams, 2001), significantly reduced the magnitude of LTP elicited by the repeated tetanization paradigm in c-fos ${ }^{\Delta \mathrm{CNS}}$ mice (with CP-101,606, $1.39 \pm 0.09, n=11$; without CP-101,606, $1.67 \pm 0.09, n=16 ; p=0.04$ ) (Fig. 5B). We did not observe a similar NR2B dependence in control animals (with CP-101,606, $1.42 \pm 0.08 ; n=15$; without CP-101,606, $1.54 \pm$ $0.08, n=16 ; p=0.32$ ) (Fig. $5 C$ ). This finding suggests that in adult wild-type animals, in which c-Fos is not disrupted, LTP is primarily induced by NR2A-type NMDA receptors.

\section{Discussion}

The presented behavioral and electrophysiological studies of mice with a CNS-specific c-fos deletion demonstrate a critical role for this immediate early gene in learning and memory. Unlike conventional c-fos knock-out mice with strong developmental impairments (Johnson et al., 1992; Wang et al., 1992; Paylor et al., 1994; Watanabe et al., 1996), the c-fos ${ }^{\Delta \mathrm{CNS}}$ mice could not be distinguished from their littermates in the home cage. Furthermore, $c-f o s{ }^{\Delta \mathrm{CNS}}$ mice were inconspicuous in locomotion, emotional behavior, and general features of water maze learning and fear conditioning, such as platform searching or toneconditioning, respectively. However, in several specific hippocampus-dependent aspects of these learning tasks, such as spatial learning (probe trial) and contextual conditioning, c-fos ${ }^{\Delta \mathrm{CNS}}$ mice exhibited significant memory deficits. These results are consistent with a study in which c-fos expression was reduced in the rat brain by antisense oligonucleotides, resulting in impaired long-term spatial learning but normal strategic learning in the water maze (Guzowski, 2002). In contrast, mice with a hippocampus-specific disruption of c-fos exhibited normal water maze learning (Zhang et al., 2002a). This could be attributable to the deletion characteristics of the floxed c-fos allele, because the hippocampal recombination rate was $95 \%$ in principal neurons but only $70 \%$ in dentate granule cells (Zhang et al., 2002b). Because of the strong and early expression of the nestin promoter, c-fos deletion is complete in c-fos ${ }^{\Delta \mathrm{CNS}}$ mice, as judged by Southern blot and immunohistochemical analysis. In addition, deletion is not restricted to the hippocampus but occurred throughout the CNS. Thus, the lack of c-Fos expression in brain areas outside of the hippocampus may have also contributed to the learning deficits found in c-fos ${ }^{\Delta \mathrm{CNS}}$ mice. Furthermore, we used a less intense training protocol with only one trial per day, which bears higher chances of detecting more subtle learning deficits than a so-called spaced protocol with several training trials per day spaced by $1 \mathrm{hr}$ intertrial intervals (Kogan et al., 1997; Gass et al., 1998).

In $c$-fos ${ }^{\Delta \mathrm{CNS}}$ mice, the deficits in spatial learning and contextual conditioning correlated with reduced LTP at hippocampal CA3-to-CA1 connections when a single tetanization procedure was applied. With four tetanizations, in contrast, the magnitude of LTP in c-fos ${ }^{\Delta \mathrm{CNS}}$ mice reached a level comparable with that in control mice. A similar restoration of LTP was observed in mice lacking either the NR2A subunit of the NMDA receptor (Sakimura et al., 1995; Kiyama et al., 1998) or the cytoplasmic C-terminal domain of the NR2A subunit (Köhr et al., 2003). This suggests that the LTP deficit in c-fos ${ }^{\Delta \mathrm{CNS}}$ mice observed with the single tetanization paradigm might result from a loss of NR2Atype signaling. In studies of mice with deficient NR2A-mediated signaling, the restoration of LTP was mediated by NR2Bsubunit-containing NMDA receptors (Sakimura et al., 1995; Kiyama et al., 1998; Köhr et al., 2003). Therefore, we hypothesized that in c-fos ${ }^{\Delta \mathrm{CNS}}$ mice, NR2B-specific inhibitors should diminish LTP restoration. Indeed, blocking NR2B-type receptors with CP-101,606 prevented the restoration of reduced LTP, indicating that the NR2A-type-induced LTP pathway is c-Fos dependent. It is possible that the receptor function is compromised because of developmental defects (e.g., in receptor localization or redistribution), or that other key players of the NR2A-typemediated pathway are missing. The lack of c-Fos may also cause a signaling impairment attributable to loss of downstream target gene expression, because the c-fos promoter is induced by tetanic stimulations, as seen by induction of green fluorescent protein fluorescence in CA1 neurons after tetanization in slices from c-fos ${ }^{\Delta \mathrm{CNS}}$ mice (data not shown). The reduced PPF is another indication that c-Fos is involved in changes in synaptic plasticity. At CA3-to-CA1 synapses, PPF is thought to be caused by an increase in the presynaptic release probability (Foster and McNaughton, 1991), so that a high and low facilitation ratio should indicate a low and high release probability, respectively (Murthy et al., 1997). We found a reduced PPF in the c-fos ${ }^{\Delta \mathrm{CNS}}$ mice.

The conclusion of a less operative NR2A pathway in the absence of c-Fos is strongly supported by the striking similarities between c-fos ${ }^{\Delta \mathrm{CNS}}$ mice and NR2A-deficient mice with respect to 


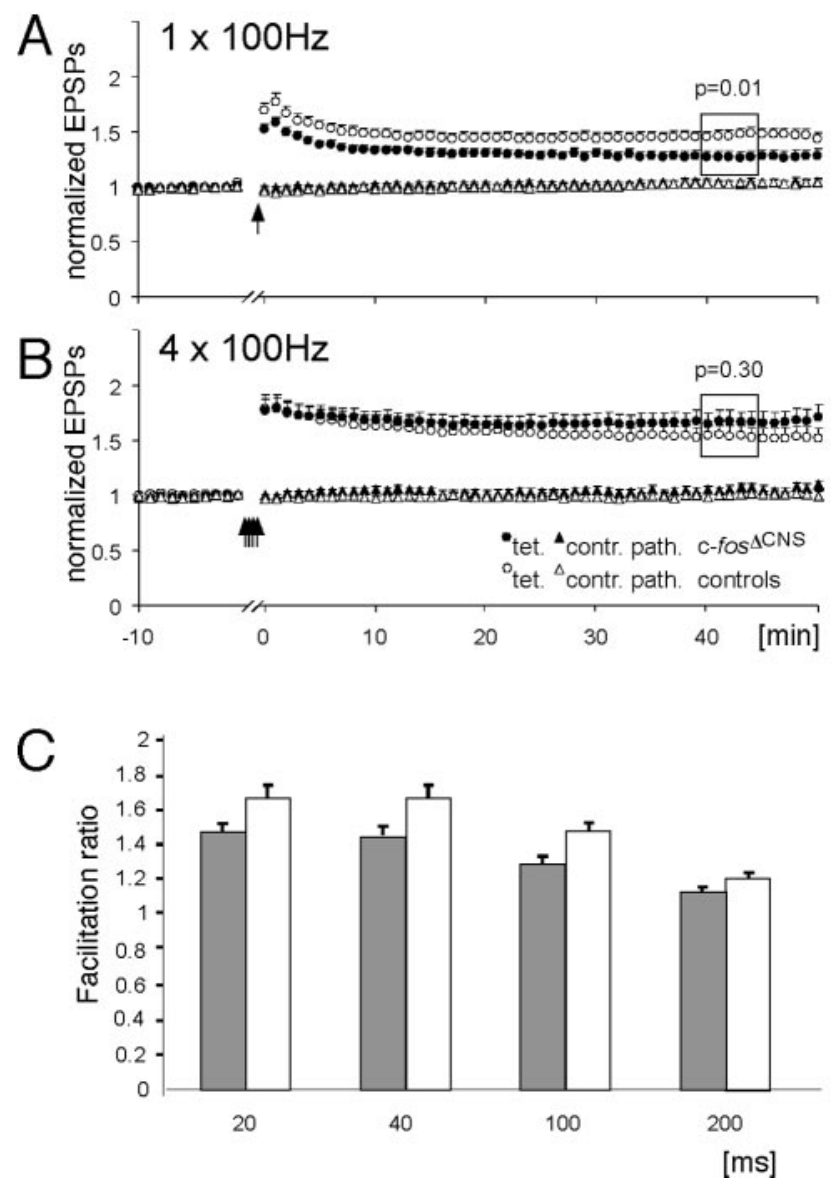

Figure 4. Long-term potentiation is impaired in c-fos ${ }^{\Delta C N S}$ mice. A, Summary graph of extracellular EPSP slopes in c-fos ${ }^{\Delta \mathrm{CNS}}$ mice (filled symbols) and control littermates (open symbols) when a single tetanization was used to induce LTP. The circles represent the tetanized pathway (tet.), and the triangles represent the untetanized control pathway (contr. path.). LTP in slices from c-fos ${ }^{\Delta \text { CNS }}$ mice (tetanized pathway, $1.27 \pm 0.05$; control pathway, $1.04 \pm 0.02 ; n=25$ ) is significantly reduced ( $p=0.01$ ) when compared with LTP in control mice (tetanized pathway, $1.46 \pm 0.05$; control pathway, $1.03 \pm 0.02 ; n=23$ ). The arrow indicates the time point of tetanic stimulation. Error bars represent SEM. $B$, As in $A$; however, data are from experiments in which a $4 \times$ tetanization paradigm was used. The period between the first and the fourth tetanization ( $15 \mathrm{~min}$ ) has been removed. The amount of $L T P$ is comparable in the two genotypes (c-fos ${ }^{\Delta \text { CNS }}$ mice, $1.67 \pm 0.09, n=16$; control littermates, $1.54 \pm 0.08, n=16$ ). C, Mean paired-pulse ratios for $c$-fos ${ }^{\Delta \mathrm{CNS}}$ (filled columns) and wild-type (open columns) mice at four interstimulus intervals $(20,50,100$, and $200 \mathrm{msec})$. At all four intervals, a slight reduction of paired-pulse facilitation is present in c-fos ${ }^{\Delta \text { CNS }}$ mice ( $p<0.05$, respectively). Error bars indicate SEM.

hippocampus-specific learning behavior (Sakimura et al., 1995; Kiyama et al., 1998). The NR2A ${ }^{-1-}$ mice also showed normal strategic but impaired spatial learning in the water maze as well as normal cue-dependent but reduced contextual fear conditioning (Sakimura et al., 1995; Kiyama et al., 1998). These learning deficits were milder than those observed in mice with complete absence of NMDA receptors in the hippocampus, which have severe learning deficits coupled with a lack of LTP (Tsien et al., 1996).

Our results suggest that NR2A-type and NR2B-type NMDA receptors activate different biochemical cascades downstream of the $\mathrm{Ca}^{2+}$ influx in vivo. Recent in vitro experiments on primary hippocampal neuronal cultures have also revealed the existence of different NMDA receptor-mediated signaling pathways (Hardingham et al., 2002). Whereas synaptically localized NR2A-type NMDA receptors triggered cellular pathways with anti-apoptotic effects, extrasynaptic NR2B-type-dependent pathways caused
A
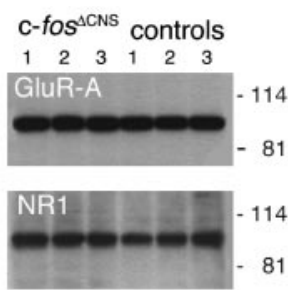

C-fos ${ }^{\Delta C N S}$ controls NR2A $A C / \Delta C$
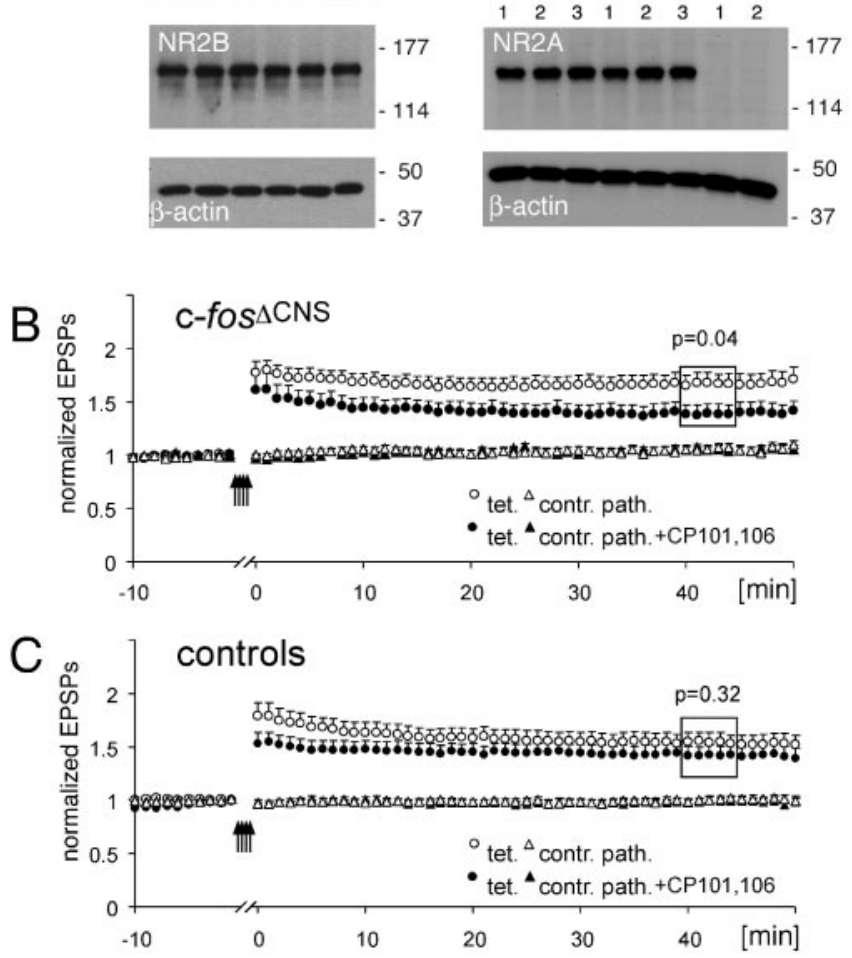

Figure 5. Restoration of LTP induction in c-fos ${ }^{\Delta \mathrm{CNS}}$ mice depends on NR2B-type NMDA receptors. $A$, Western blot experiments with hippocampal protein extracts demonstrate regular expression levels of the NMDA receptor subunits NR1, NR2A, and NR2B, as well as of the AMPA receptor subunit GluRA. Protein extracts from three different $c-f 0 s{ }^{\Delta \mathrm{CNS}}$ and three control mice were analyzed, respectively. Extracts from NR2A ${ }^{\Delta C / \Delta C}$ mice, which lack the C-terminal epitope recognized by the NR2A antibody, served as a control for the specificity of the antibody reaction. Molecular masses are indicated in kilodaltons on the right, and $\beta$-actin levels show that similar amounts of total protein were used. $B$, Summary graph of extracellular EPSP slopes in c-fos ${ }^{\triangle C N S}$ mice in the presence (filled symbols) and absence (open symbols) of the NR2B blocker (P$101,606(10 \mu \mathrm{M})$. The circles represent the tetanized pathway (tet.), and the triangles represent the untetanized control pathway (contr. path.). A $4 \times$ tetanization procedure was used to induce LTP. The magnitude of LTP is reduced in the presence of CP-101,606 (tetanized pathway with (P-101,606, 1.39 $\pm 0.09, n=11$; tetanized pathway without CP-101,606, 1.67 \pm 0.09 , $n=16 ; p=0.04)$. Error bars represent SEM. Arrows indicate time points of tetanic stimulation. The period between the first and the fourth tetanization ( $15 \mathrm{~min}$ ) has been removed. C, As in A; however, from control animals. The magnitude of LTP in control animals is unchanged in the presence of CP-101,606 (tetanized pathway with CP-101,606, 1.42 $\pm 0.08, n=15$; tetanized pathway without (P-101,606, $1.54 \pm 0.08, n=16 ; p=0.32)$.

cAMP response element-binding protein shut-off and cell death (Hardingham et al., 2002). None of the mouse models with blocked NR2A-type pathways $\left(\mathrm{NR} 2 \mathrm{~A}^{-/-}, \mathrm{NR} 2 \mathrm{~A}^{\Delta \mathrm{C} / \Delta \mathrm{C}}\right)$ or c-fos ${ }^{\Delta \mathrm{CNS}}$ mice exhibited increased neuronal death under physiological conditions (Sakimura et al., 1995; Köhr et al., 2003). In contrast, in mice with a hippocampus-specific c-fos disruption, pathological excitation by kainic acid induced a significant increase in neuronal apoptosis, probably caused by a dysregulation of GluR6 and brain-derived neurotrophic factor (Zhang et al., 2002 b). Our results suggest that increased apoptosis in the absence of c-Fos may also be influenced by a reduced protective 
effect of the NR2A-mediated pathway, which is unable to balance the apoptotic pathway mediated by the activation of NR2B-type NMDA receptors (Hardingham et al., 2002).

In this study, the molecular details of specific pathway activation by different NMDA receptor subtypes remain unclear. Köhr et al. (2003) observed that NR2A-type receptors, which do not contain the large intracellular NR2A C-terminal domain, provide sufficient synaptic $\mathrm{Ca}^{2+}$ signaling but fail to activate the NR2Atype pathways during LTP induction. Both $c-f o s^{\Delta \mathrm{CNS}}$ and $\mathrm{NR} 2 \mathrm{~A}^{\Delta \mathrm{C} / \Delta \mathrm{C}}$ mice indicate a physiological relevance for different, selective NMDA receptor subtype-activated pathways in vivo.

\section{References}

Andersen P (1960) Interhippocampal impulses. II. Apical dendritic activation of CA1 neurons. Acta Physiol Scand 48:178-208.

Bannerman DM, Good MA, Butcher SP, Ramsay M, Morris RG (1995) Distinct components of spatial learning revealed by prior training and NMDA receptor blockade. Nature 378:182-186.

Chiu R, Boyle WJ, Meek J, Smeal T, Hunter T, Karin M (1988) The c-Fos protein interacts with c-Jun/AP-1 to stimulate transcription of AP-1 responsive genes. Cell 54:541-552.

Dymecki SM (1996) Flp recombinase promotes site-specific DNA recombination in embryonic stem cells and transgenic mice. Proc Natl Acad Sci USA 93:6191-6196.

Foster TC, McNaughton BL (1991) Long-term enhancement of CA1 synaptic transmission is due to increased quantal size, not quantal content. Hippocampus 1:79-91.

Gass P, Wolfer DP, Balschun D, Rudolph D, Frey U, Lipp HP, Schutz G (1998) Deficits in memory tasks of mice with CREB mutations depend on gene dosage. Learn Mem 5:274-288.

Greenberg ME, Ziff EB (1984) Stimulation of 3T3 cells induces transcription of the c-fos proto-oncogene. Nature 311:433-438.

Guzowski JF (2002) Insights into immediate-early gene function in hippocampal memory consolidation using antisense oligonucleotide and fluorescent imaging approaches. Hippocampus 12:86-104.

Halazonetis TD, Georgopoulos K, Greenberg ME, Leder P (1988) c-Jun dimerizes with itself and with c-Fos, forming complexes of different DNA binding affinities. Cell 55:917-924.

Hardingham GE, Fukunaga Y, Bading H (2002) Extrasynaptic NMDARs oppose synaptic NMDARs by triggering CREB shut-off and cell death pathways. Nat Neurosci 5:405-414.

Herdegen T, Leah JD (1998) Inducible and constitutive transcription factors in the mammalian nervous system: control of gene expression by Jun, Fos and Krox, and CREB/ATF proteins. Brain Res Brain Res Rev 28:370-490.

Hollmann M, Heinemann S (1994) Cloned glutamate receptors. Annu Rev Neurosci 17:31-108.

Johnson RS, Spiegelman BM, Papaioannou V (1992) Pleiotropic effects of a null mutation in the c-fos proto-oncogene. Cell 71:577-586.

Kandel ER (2001) The molecular biology of memory storage: a dialogue between genes and synapses. Science 294:1030-1038.

Katz B, Miledi R (1968) The role of calcium in neuromuscular facilitation. J Physiol (Lond) 195:481-492.

Kiyama Y, Manabe T, Sakimura K, Kawakami F, Mori H, Mishina M (1998) Increased thresholds for long-term potentiation and contextual learning in mice lacking the NMDA-type glutamate receptor $\epsilon 1$ subunit. J Neurosci 18:6704-6712.
Kogan JH, Frankland PW, Blendy JA, Coblentz J, Marowitz Z, Schutz G, Silva AJ (1997) Spaced training induces normal long-term memory in CREB mutant mice. Curr Biol 7:1-11.

Köhr G, Jensen V, Koester H, Mihaljevic ALA, Utvik JK, Kvello A, Ottersen OP, Seeburg PH, Sprengel R, Hvalby O (2003) Intracellular domains of NMDA receptor subtypes are determinants for LTP induction. J Neurosci, in press.

Kouzarides T, Ziff E (1988) The role of the leucine zipper in the fos-jun interaction. Nature 336:646-651.

Martin SJ, Grimwood PD, Morris RG (2000) Synaptic plasticity and memory: an evaluation of the hypothesis. Annu Rev Neurosci 23:649-711.

Morris RG, Garrud P, Rawlins JN, O’Keefe J (1982) Place navigation impaired in rats with hippocampal lesions. Nature 297:681-683.

Muller R, Tremblay JM, Adamson ED, Verma IM (1983) Tissue and cell type-specific expression of two human c-onc genes. Nature 304:454-456.

Murthy VN, Sejnowski TJ, Stevens CF (1997) Heterogeneous release properties of visualized individual hippocampal synapses. Neuron 18:599-612.

Nicoll RA, Kauer JA, Malenka RC (1988) The current excitement in longterm potentiation. Neuron 1:97-103.

Paylor R, Johnson RS, Papaioannou V, Spiegelman BM, Wehner JM (1994) Behavioral assessment of c-fos mutant mice. Brain Res 651:275-282.

Rogan MT, LeDoux JE (1996) Emotion: systems, cells, synaptic plasticity. Cell 85:469-475.

Sakimura K, Kutsuwada T, Ito I, Manabe T, Takayama C, Kushiya E, Yagi T, Aizawa S, Inoue Y, Sugiyama H, Mishina M (1995) Reduced hippocampal LTP and spatial learning in mice lacking NMDA receptor epsilon 1 subunit. Nature 373:151-155.

Stocca G, Vicini S (1998) Increased contribution of NR2A subunit to synaptic NMDA receptors in developing rat cortical neurons. J Physiol (Lond) 507:13-24

Timpl P, Spanagel R, Sillaber I, Kresse A, Reul JM, Stalla GK, Blanquet V, Steckler T, Holsboer F, Wurst W (1998) Impaired stress response and reduced anxiety in mice lacking a functional corticotropin-releasing hormone receptor 1. Nat Genet 19:162-166.

Tischmeyer W, Grimm R (1999) Activation of immediate early genes and memory formation. Cell Mol Life Sci 55:564-574.

Tronche F, Kellendonk C, Kretz O, Gass P, Anlag K, Orban PC, Bock R, Klein R, Schutz G (1999) Disruption of the glucocorticoid receptor gene in the nervous system results in reduced anxiety. Nat Genet 23:99-103.

Tsien JZ, Huerta PT, Tonegawa S (1996) The essential role of hippocampal CA1 NMDA receptor-dependent synaptic plasticity in spatial memory. Cell 87:1327-1338.

Wang ZQ, Ovitt C, Grigoriadis AE, Mohle-Steinlein U, Ruther U, Wagner EF (1992) Bone and haematopoietic defects in mice lacking c-fos. Nature 360:741-745.

Watanabe Y, Johnson RS, Butler LS, Binder DK, Spiegelman BM, Papaioannou VE, McNamara JO (1996) Null mutation of c-fos impairs structural and functional plasticities in the kindling model of epilepsy. J Neurosci $16: 3827-3836$.

Williams K (2001) Ifenprodil, a novel NMDA receptor antagonist: site and mechanism of action. Curr Drug Targets 2:285-298.

Zhang J, McQuade JM, Vorhees CV, Xu M (2002a) Hippocampal expression of c-fos is not essential for spatial learning. Synapse 46:91-99.

Zhang J, Zhang D, McQuade JS, Behbehani M, Tsien JZ, Xu M (2002b) c-fos regulates neuronal excitability and survival. Nat Genet 30:416-420.

Zucker RS (1989) Short-term synaptic plasticity. Annu Rev Neurosci 12:13-31. 